\title{
Estrategias de aprendizaje en la virtualidad y la satisfacción de los servicios Universitarios: una revisión Literaria
}

Virtuality learning strategies and the satisfaction of university services: a Literary review

DOI: https://doi.org/10.33262/rmc.v6i2.1087

Florcita Janeth Arellano Espinoza ${ }^{1}$

Universidad Estatal de Bolívar

https://orcid.org/0000-0002-4325-9317

farellano@ueb.edu.ec

Mayra Cecilia Coello Villa ${ }^{2}$

Universidad Estatal de Bolívar

https://orcid.org/0000-0002-4139-4064

mcoello@ueb.edu.ec

Manolo Javier Vásconez Torres ${ }^{3}$

Universidad Estatal de Bolívar

(iD https://orcid.org/0000-0003-4052-6843

mvasconez@ueb.edu.ec

Nicolás Alexander Ladino Pazmiño ${ }^{4}$

Universidad Estatal de Bolívar

iD https://orcid.org/0000-0002-9090-9343

nladino@ueb.edu.ec

\section{RESUMEN}

El objetivo de esta investigación fue analizar la aplicación de las estrategias de aprendizaje y el estado de satisfacción de los estudiantes sobre los servicios universitarios, el método aplicado fue una revisión de la literatura. Los resultados principales fueron que las estrategias más aplicadas en la virtualidad son las estrategias metacognitivas, la autorregulación y las estrategias de procesamiento y uso de la información, por otra parte, los estudiantes basan su satisfacción principalmente en la planta docente, sin embargo, también se destacan aspectos como la infraestructura, capacidad de servicio, comunicación y competencias y actitudes de los docentes. Se concluye, que los servicios de las universidades fueron moderados desde la perspectiva de los estudiantes, esto significa que las universidades tienen la capacidad suficiente para seguir mejorando. Además, se identificó que las actividades académicas son más importantes involucrando tanto a las estrategias de aprendizaje como la satisfacción. 
PALABRAS CLAVE: Aulas virtuales; Educación superior; Estrategias de aprendizaje; Satisfacción; Servicios universitarios

\begin{abstract}
The purpose of this research was to analyze the application of learning strategies and the state of satisfaction of students about university services, the applied method was a literature review. The main results were that the most applied strategies in virtuality are metacognitive strategies, self-regulation and information processing and use strategies, on the other hand, students base their satisfaction mainly on the teaching staff, however, they also Aspects such as infrastructure, service capacity, communication and skills and attitudes of teachers stand out. It is concluded that the services of the universities were moderate from the students' perspective, this means that the universities have sufficient capacity to continue improving. In addition, it was identified that academic activities are more important involving both learning strategies and satisfaction.
\end{abstract}

KEYWORDS: Higher education; Learning strategies; Satisfaction; University services; Virtual classrooms

\title{
INTRODUCCIÓN
}

Las universidades son consideradas las principales instituciones responsables de la producción y democratización de conocimientos, sino tenemos una buena educación, el Ecuador no saldrá del subdesarrollo, por tanto, determinan el futuro de las naciones. Las universidades como instituciones de educación superior públicas tienen que lidiar con una mayor competencia para los estudiantes, menor financiamiento gubernamental y presiones crecientes para los resultados de la investigación, entre otros problemas (Sabateyana, Saduov, Madiyarova, Jempeissova, Selezneva, \& Fursova, 2018).

La globalización en la educación ha fomentado una mayor competencia entre las universidades, en la actualidad se están dando cuenta de la importancia de las expectativas de las partes interesadas en su proceso de toma de decisiones estratégicas. En un entorno cada vez más competitivo, mejorar y mantener una ventaja competitiva es una tarea difícil y se vuelve importante estudiar a fondo los factores que permiten que las IES atraigan y retengan a los estudiantes, especialmente considerando las muchas opciones que se les ofrecen (P, Sembiring, S, Sembiring, Taringa \& Sembiring, 2017). 
El perfil humano al que quieren llegar las universidades, es el perfil que tiene una alta capacidad de aprendizaje y es responsable de su propio aprendizaje. En este sentido, adquiere importancia la capacidad de utilizar estrategias de aprendizaje que reflejen las características individuales de los estudiantes (Pedro, Mendez y Leurenco, 2018)., hay muchos factores que contribuyen al éxito de los estudiantes y los Sin embargo más importantes son saber aprender, gestionar el tiempo, leer y escuchar de forma eficaz, tomar notas eficaces, comprender y recordar. Las estrategias de aprendizaje están direccionado a los comportamientos de los alumnos, que están destinados a influir en cómo procesar la información, se conoce seis características de las estrategias de aprendizaje: procedimentales, intencionadas, esforzadas, voluntarias, esenciales y facilitadoras (Pegalajar, 2016).

La educación es un proceso de cambio, y en este proceso, la satisfacción de los estudiantes con la educación que reciben y la institución donde estudian es muy esencial para la continuación efectiva del proceso (Contreras, Pérez, Hechenleitner, Vaccarezza y Toirkeins, 2019). Esto se debe a que, la percepción de la calidad en servicios como la educación y la satisfacción del cliente generalmente se evalúa observando el lugardonde se brinda el servicio, el personal que brinda el servicio, el funcionamiento institucional y las personas que reciben el servicio (Kasalak \& Dagyar, 2020).

La satisfacción de los estudiantes indica lo que sienten de una universidad con respecto al profesorado, servicios de consultoría, servicios de gestión, recursos, instalaciones informáticas y cursos, y planes de estudios en la universidad. Es difícil inferir, hasta qué punto el nivel de satisfacción de los estudiantes refleja la calidad de la educación. Entre los muchos factores que contribuyen a la satisfacción (incluidos los aspectos académicos, sociales, personales, administrativos, financieros, materiales y ambientales), que son diversos y cambian según el contexto (Abella, Ausín, Delgado, Hortiguela y Solano, 2018).

Se ha llevado a cabo una extensa investigación en el sector de la Educación, estudiando los factores que pueden afectar la satisfacción de los estudiantes, la buena calidad de la educación brinda mejores oportunidades de aprendizaje. Los niveles de satisfacción o insatisfacción afectan fuertemente el éxito o el fracaso del aprendizaje del estudiante, dichos factores incluyen el rendimiento, el rendimiento de los profesores, el entorno del aula, las instalaciones de aprendizaje y la reputación de la institución. Actualmente, en la academia se han enfocado en identificar y satisfacer las necesidades y expectativas de los 
estudiantes, asignando recursos, reformando las políticas públicas, capacitaciones, actualización de conocimientos, sin embargo, la virtualidad tiene que manejarse desde una visión bilateral, es decir, no solo esperar incentivos de la academia, más bien, es necesario que los estudiantes de forma autónoma intervengan en el proceso.

Es frente a las adversidades suscitadas en el año 2020 muchas de las actividades se han visto en la necesidad de modificar sus prácticas, tal es el caso de la educación, ha obligado que la transición hacia a un nuevo modelo educativo (Rojo y Bonilla, 2020). El propósito de esta investigación es analizar la aplicación de las estrategias de aprendizaje y el estado de satisfacción de los estudiantes sobre los servicios universitarios del año 2014 hasta el 2020. En esta revisión de literatura, se presenta y se discute definiciones de estrategias de aprendizaje y de satisfacción universitaria comparando diferentes resultados. Finalmente, luego de identificar las estrategias más comunes que aplican los estudiantes en diferentes formatos en la educación superior, también se analizó qué aspectos son los primordiales en la satisfacción que le genera al estudiante.

\section{DESARROLLO}

\section{Estrategias de aprendizaje}

Este estudio toma como punto de partida, la comprensión de las estrategias de aprendizaje como: procedimientos que consisten en una combinación de pasos, o secuencias de acción, que el estudiante elige seguir para tener una experiencia de aprendizaje más significativa y para resolver problemas y satisfacer las demandas académicas. En este contexto, se sabe que las estrategias de aprendizaje son medios que los estudiantes parecen emplear para mejorar la efectividad de su aprendizaje (P. López, Gallegos, Vilca y López, 2018), y Gozalo, Leon y Mendo (2020), los ve como operaciones, mecanismos, tácticas y procedimientos mentales que se utilizan de manera más o menos consciente para realizar tareas comunicativas o para afrontar nuevas situaciones de aprendizaje.

Además, se conoce, que también son acciones específicas tomadas por el alumno para hacer que el aprendizaje sea más fácil, más rápido, más agradable, más autodirigido y más transferible a nuevas situaciones (Vera, Poblete y Días, 2018). Las estrategias de aprendizaje a nivel universitario están centradas en el estudiante e implican enfocar todo el proceso de base del conocimiento, que, dada la madurez del aprendiz, es mucho más activo y autónomo. En este proceso, los estudiantes regulan su aprendizaje y seleccionan sus estrategias, el aprendizaje autorregulado está orientado a procesos en los que los 
estudiantes dirigen sus operaciones, tendencias y emociones de manera metódica y controlada, hacia el logro de sus metas planteadas (Montero, Sierra y Mendiarrieta, 2017).

Alarcón, Zapata, Alarcón, Natividad y Rodríguez (2019) afirma que la autorregulación está estrechamente ligada a las variables motivacionales que la influyen, como el establecimiento de metas, la autoeficacia, las expectativas, la actitud adoptada hacia la meta y el rendimiento académico posterior. Asimismo, uno de los principales objetivos de las universidades, en general, es que los estudiantes sean capaces de controlar su proceso de aprendizaje y adquirir competencias que puedan utilizar dentro y fuera del ámbito del aula, y el eje central es el aprendizaje autónomo que les ayudará para vida (Hoffman, Ledesma y Liporace, 2017). De esta manera, se puede mencionar también al uso de la tecnología como un factor esencial con relación al contexto del uso, sobre todo como medio de aplicación para estrategias de aprendizaje ya que en países desarrollados han sido usadas como una herramienta efectiva en la educación ya que vincula al docente con el desarrollo de cualidades investigativas (Mora, Bonilla, Nuñez y Sarmiento, 2018).

\section{Satisfacción del estudiante con los servicios universitarios}

La satisfacción es un sentimiento de felicidad que se obtiene cuando una persona satisface sus necesidades y deseos. Es un estado que siente una persona que ha experimentado un desempeño o un resultado que cumplió con las expectativas que tenía de ella, en consecuencia, la satisfacción se puede definir como una experiencia de cumplimiento de los resultados esperados.

La satisfacción de los estudiantes como actitud a corto plazo, es el resultado de una evaluación de las experiencias educativas de los estudiantes, es un antecedente positivo de la lealtad de los estudiantes y es el resultado de un sistema educativo, la satisfacción de los estudiantes se mide a través del grado de conocimientos que adquieren en las aulas (A. Lara, N. Lara, G Lara y Bonilla, 2018). Así como también, Cadena, Mejías, Vega y Vásquez (2015) definen la satisfacción del estudiante como la disposición de los estudiantes mediante la evaluación subjetiva de los resultados educativos y la experiencia. Por lo tanto, la satisfacción del estudiante se puede definir en función del nivel relativo de experiencias y desempeño percibido sobre el servicio educativo durante el período de estudio. Al considerarlos todos, la satisfacción de los estudiantes puede definirse como 
una actitud a corto plazo resultante de una evaluación de la experiencia educativa, los servicios y las instalaciones de los estudiantes.

La satisfacción de los estudiantes es un proceso multidimensional en el que influyen diferentes factores. Según Dos Anjos \& Dos Reis (2019), el promedio de puntos de calificación es el factor más influyente en la satisfacción del estudiante. Salinda \& Lalitha (2017) identificaron dos grupos de influencias sobre la satisfacción de los estudiantes en la educación superior como factores personales e institucionales. Los factores personales cubren la edad, el género, el empleo, el estilo de aprendizaje preferido, el puntaje de calificación del estudiante y los factores institucionales cubren la calidad de las instrucciones, la rapidez de la retroalimentación del instructor, la claridad de las expectativas y el estilo de enseñanza. Tumino \& Poitevin (2014) identificaron la calidad de los profesores, la calidad de las instalaciones físicas y el uso eficaz de la tecnología como factores determinantes clave de la satisfacción de los estudiantes. Además, la satisfacción de los estudiantes en las universidades está muy influenciada por la calidad del salón de clases, la calidad de la retroalimentación, la relación profesor-alumno, la interacción con los compañeros, el contenido del curso, el equipo de aprendizaje disponible, las instalaciones de la biblioteca y los materiales de aprendizaje (Peñaherrera et al., 2019). Además de eso, la capacidad de enseñanza, el plan de estudios flexible, el estatus y el prestigio universitario, la independencia, el cuidado del profesorado, el crecimiento y desarrollo de los estudiantes, el enfoque en el estudiante, el clima del campus, la efectividad institucional y las condiciones sociales se han identificado como determinantes principales.

Ahora, se busca estudiar para llenar una investigación brecha y ofrecer una mayor comprensión de los elementos que se han asociado fuertemente con la satisfacción general de los estudiantes, por eso, se toma en consideración el modelo conceptual para la satisfacción del estudiante adaptado del modelo I-E-O de Astin. 


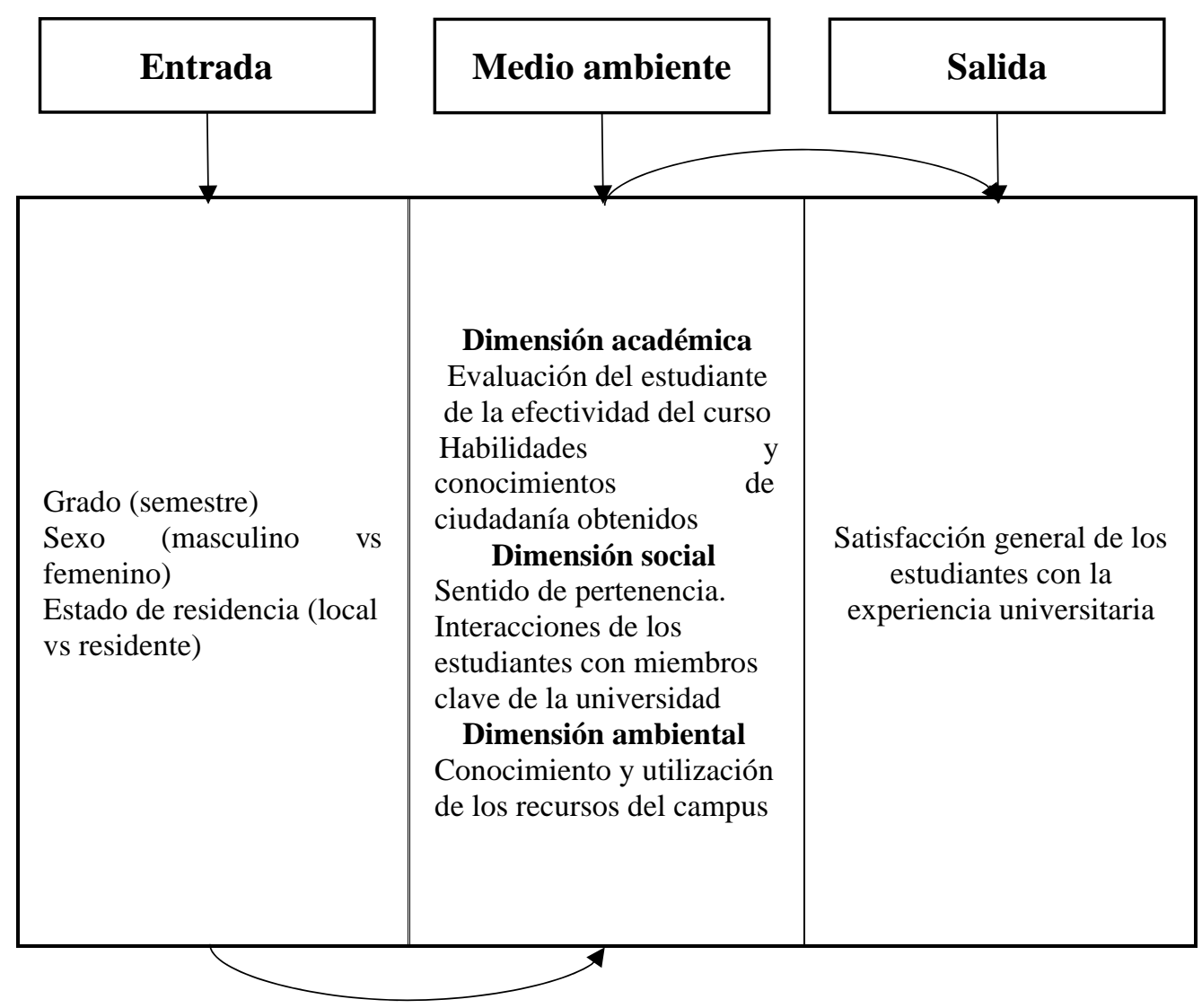

Figura 1. Modelo I-E-O de Astin

Fuente: Información obtenida de "Investigating Determinants of Student Satisfaction in the First Year of College in a Public University in the State of Qatar", (Al-Sheeb et al., 2018).

\section{MATERIALES Y METODOS}

La búsqueda de literatura en la que se basa la presente revisión, tiene el propósito de identificar artículos que puedan contribuir a dar respuesta a la investigación.

En febrero de 2021 se llevó a cabo una búsqueda sistemática en las bases de datos como: Scielo, Elsevier, EBSCO, Research Gate, Dialnet y Scopus, utilizando criterios de búsqueda como: calidad y servicios universitarios, satisfacción de servicios universitarios, importancia de servicios universitarios, estrategias de aprendizaje, estrategias educativas universitarias, proceso de aprendizaje y estudiantes universitarios. Para garantizar que los últimos hallazgos se presenten en la revisión, la búsqueda sistemática se restringió a los artículos publicados entre 2014-2021.

La búsqueda en las bases de datos generó un total de 123 documentos. El proceso inicia al realizar una revisión sistemática de los artículos completos, se categorizo y, finalmente, 
se seleccionaron 60 artículos como relevantes y se agruparon en 6 categorías principales, aspectos que fueron especificados en los criterios de inclusión. Las 6 categorías se redujeron aún más a 3 categorías basadas en una estimación de qué categorías eran las más dominantes, es decir, las categorías que implicaban el mayor número de aciertos. Por lo tanto, la revisión se basa en un total de 36 artículos y aborda las siguientes categorías: estrategias de aprendizaje, satisfacción de los servicios universitarios y la relación existente entre las estrategias de aprendizaje y satisfacción de los servicios universitarios. Se publican artículos del año 2014 (3 artículos), 2015 (3 artículos), 2016 (3 artículos), 2017 (8 artículos), 2018 (9 artículos), 2019 (6 artículos) y 2020 (4 artículos) para todo el sustento investigativo, no obstante, se utilizó 20 estudios para dar respuesta al objetivo de estudio, dicha información fue planteada en el apartado de resultados.

\section{RESULTADOS}

Se establece un enfoque global sobre la aplicación de las estrategias de aprendizaje en estudiantes universitarios.

Tabla 1. Estrategias de aprendizaje

\begin{tabular}{|c|c|c|c|c|c|}
\hline \# & Estudio & Año & $\begin{array}{l}\text { Población } \\
\text { evaluada }\end{array}$ & Estrategias & $\%$ \\
\hline 1 & $\begin{array}{lr}\text { Estrategias } & \text { de } \\
\text { Aprendizaje } & \text { y } \\
\text { Rendimiento } & \\
\text { Académico } & \text { En } \\
\text { Estudiantes } & \\
\text { Universitarios } & \text { de } \\
\text { Pregrado (Poce } & \text { \& } \\
\text { Gamarra, 2014) } & \end{array}$ & 2014 & 41 estudiantes & $\begin{array}{l}\text { - Estrategias de } \\
\text { aprendizaje cognitivas } \\
\text { - Estrategias de control de } \\
\text { recursos } \\
\text { - Estrategias } \\
\text { metacognitivas }\end{array}$ & - \\
\hline 2 & $\begin{array}{ll}\text { Evaluación } & \text { de } \\
\text { estrategias } & \text { de } \\
\text { aprendizaje } & \text { en } \\
\text { estudiantes } & \\
\text { universitarios y su } \\
\text { relación con } & \text { el } \\
\text { rendimiento } & \\
\text { académico } & \text { en }\end{array}$ & 2015 & 653 estudiantes & - Autorregulación & 56 \\
\hline
\end{tabular}




\begin{tabular}{|c|c|c|c|c|c|}
\hline & $\begin{array}{l}\text { procesos mediados } \\
\text { por TIC (Mena et al., } \\
2015 \text { ) }\end{array}$ & & & & \\
\hline 3 & $\begin{array}{l}\text { Estrategias de } \\
\text { aprendizaje en } \\
\text { alumnado } \\
\text { universitario para la } \\
\text { formación presencial } \\
\text { y } \\
\text { semipresencial } \\
\text { (Pegalajar, 2016). }\end{array}$ & 2016 & 80 estudiantes & $\begin{array}{l}\text { - Estrategias de } \\
\text { procesamiento y uso de la } \\
\text { información } \\
\text { - Estrategias } \\
\text { metacognitivas }\end{array}$ & 19 \\
\hline 4 & $\begin{array}{lr}\text { Estratégias } & \mathrm{de} \\
\text { aprendizagem } & \mathrm{em} \\
\text { universitários: } & \\
\text { Avaliação } & \mathrm{e} \\
\text { intervenção } \\
\text { (Mohallem, 2016). }\end{array}$ & 2016 & 109 estudiantes & $\begin{array}{l}\text { Estrategias de } \\
\text { aprendizaje } \\
\text { - Autorregulación } \\
\text { cognitiva metacognitiva. } \\
\text { - Autorregulación de } \\
\text { recursos internos y } \\
\text { contextuales. } \\
\text { - Autorregulación social. } \\
\text { Auto eficiencia académica } \\
\text { - Autoeficacia en la } \\
\text { regulación de formación. } \\
\text { - Autoeficacia en la } \\
\text { interacción social. } \\
\text { - Autoeficacia en acciones } \\
\text { proactivas. Autoeficacia en la } \\
\text { gestión académica. }\end{array}$ & 8.02 \\
\hline 5 & $\begin{array}{l}\text { Estilos y estrategias } \\
\text { de aprendizaje en } \\
\text { estudiantes } \\
\text { universitarios de } \\
\text { Buenos Aires }\end{array}$ & 2017 & 438 estudiantes & $\begin{array}{c}\text { - Habilidades para } \\
\text { jerarquizar información }\end{array}$ & 44.58 \\
\hline
\end{tabular}




\begin{tabular}{|c|c|c|c|c|c|}
\hline & $\begin{array}{l}\text { (Hoffman et al., } \\
\text { 2017) }\end{array}$ & & & & \\
\hline 6 & $\begin{array}{l}\text { Estrategias } \\
\begin{array}{l}\text { aprendizaje } \\
\text { estudiantes }\end{array} \\
\text { universitarios de } \\
\text { ciencias sociales: un } \\
\text { estudio empírico en } \\
\text { la escuela profesional } \\
\text { de sociología UNAP } \\
\text { (López et al., 2018) }\end{array}$ & 2018 & 217 estudiantes & $\begin{array}{l}\text { Aprendizaje autónomo } \\
\text { - Motivación interna } \\
\text { - Valoración de la tarea } \\
\text { - Autorregulación } \\
\text { - Elaboración }\end{array}$ & $\begin{array}{r}93.1 \\
92.6 \\
89.9 \\
91.7\end{array}$ \\
\hline 7 & $\begin{array}{lrr}\text { Empleo de } & \text { las } \\
\text { estrategias } & & \text { de } \\
\text { aprendizaje en } & \text { la } \\
\text { universidad. } & \text { Un } \\
\text { estudio de } & \text { caso } \\
\text { (Alarcón et al.,2019). }\end{array}$ & 2019 & 13 estudiantes & $\begin{array}{l}\text { - } \text { Estrategias } \\
\text { metacognitivas } \\
\text { - Procesamiento de la } \\
\text { información } \\
\text { - Control del contexto }\end{array}$ & - \\
\hline 8 & $\begin{array}{lr}\text { Estrategias } & \text { de } \\
\text { aprendizaje } & \text { y } \\
\text { desempeño } & \\
\text { académico } & \text { en } \\
\text { estudiantes } & \text { de } \\
\text { Medicina (Salazar \& } \\
\text { Heredia, 2019) }\end{array}$ & 2019 & 135 estudiantes & $\begin{array}{l}\text { - Manejo de los recursos } \\
\text { de información }\end{array}$ & - \\
\hline
\end{tabular}

Fuente: Elaborado por los autores

Las estrategias de aprendizaje describen las preferencias de los estudiantes durante las actividades de aprendizaje que involucran el incorporar y conciliar exitosamente información novedosa con la preexistente. En la enseñanza universitaria las estrategias de aprendizaje juegan un papel fundamental, su carácter intencional permite trazar un plan de acción de modo tal que, a partir de la información que se desea incorporar, sea posible seleccionar aquellas conductas que faciliten la tarea, todo esto justificándose en los datos encontrados por medio de la revisión bibliográfica. 


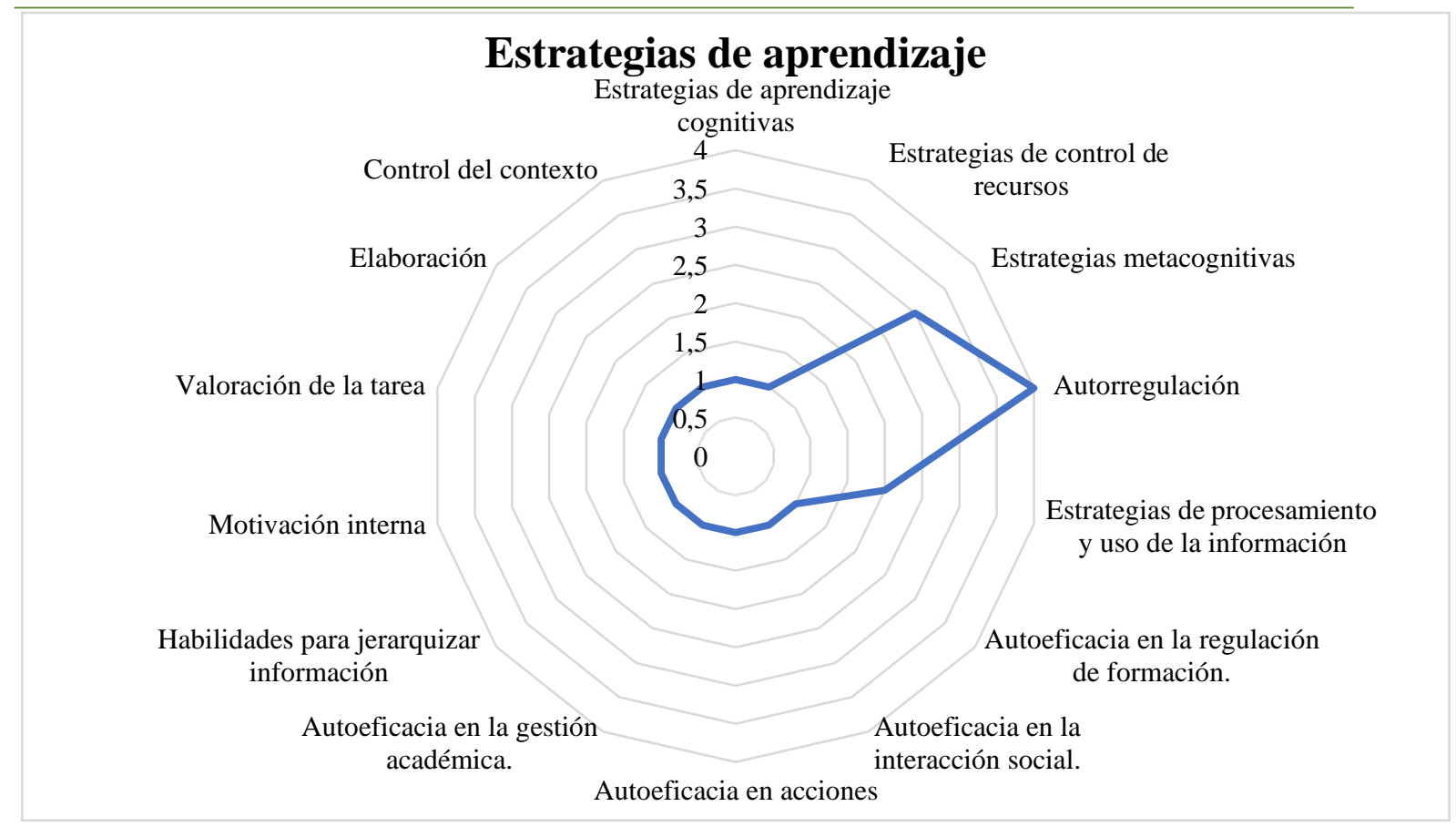

Figura 2. Estrategias de aprendizaje

Fuente: Elaborado por los autores

El uso de estrategias de aprendizaje constituye un fuerte factor protector para el éxito académico, contribuyendo en gran medida a la mejor adaptación de los estudiantes a la Educación Superior. Los resultados revelaron que los estudiantes aplican las estrategias de aprendizaje, entre las más comunes se encuentra las estrategias metacognitivas, la autorregulación y las estrategias de procesamiento y uso de la información, es necesario mencionar que gran representación de la autorregulación se ha centrado en la cognitiva metacognitiva, recursos internos y contextuales y aspectos sociales. Los datos se discuten en términos no solo de la importancia de las estrategias de aprendizaje para fortalecer la adaptación y el éxito académico del estudiante universitario, sino también su relevancia.

Tabla 2. Satisfacción de los servicios universitarios

\begin{tabular}{|c|c|c|c|c|c|}
\hline \# & Estudio & Año & $\begin{array}{c}\text { Población } \\
\text { evaluada }\end{array}$ & Aspectos evaluados & $\%$ \\
\hline 1 & $\begin{array}{l}\text { Estudio de la } \\
\text { Satisfacción de los } \\
\text { Estudiantes con los } \\
\text { Servicios } \\
\text { Educativos }\end{array}$ & 2014 & $\begin{array}{ll}\text { Estudio de } \\
\text { validez }\end{array}$ & $\begin{array}{l}\text { Altos niveles de } \\
\text { satisfacción } \\
\text { • Habilidad para la } \\
\text { enseñanza de los } \\
\text { Docentes. }\end{array}$ & - \\
\hline
\end{tabular}




\begin{tabular}{|c|c|c|c|c|c|}
\hline & $\begin{array}{l}\text { brindados por } \\
\text { Instituciones de } \\
\text { Educación Superior } \\
\text { del Valle de Toluca } \\
\text { (Botello et al., } \\
\text { 2014). }\end{array}$ & & & $\begin{array}{l}\text { - Nivel de } \\
\text { Autorrealización de } \\
\text { Estudiantes. } \\
\text { - Servicios } \\
\text { Administrativos. }\end{array}$ & \\
\hline 2 & $\begin{array}{l}\text { Percepción de los } \\
\text { estudiantes sobre la } \\
\text { calidad de las } \\
\text { universidades } \\
\text { privadas } \\
\text { Monterrey } \\
\text { (Alvarado et al., } \\
\text { 2015). }\end{array}$ & 2015 & 487 estudiantes & $\begin{array}{l}\text { - Desarrollo integral } \\
\text { - Componente físico } \\
\text { - Planta docente } \\
\text { - Habilidad docente }\end{array}$ & $\begin{array}{l}77.3 \\
37.4 \\
10.1 \\
5.8\end{array}$ \\
\hline 3 & $\begin{array}{lr}\text { Procesos } & \text { y } \\
\text { servicios } & \\
\text { universitarios: } & \mathrm{La} \\
\text { percepción } & \mathrm{de} \\
\text { estudiantes (Ríos et } \\
\text { al., 2017) }\end{array}$ & 2017 & $\begin{array}{l}\text { Estudio de } \\
\text { validez }\end{array}$ & $\begin{array}{l}\text { - Proceso de ingreso } \\
\text { - Método de } \\
\text { enseñanza } \\
\text { aprendizaje } \\
\text { - Evaluaciones } \\
\text { - Organización de las } \\
\text { carreras } \\
\text { - Equipo docente } \\
\text { - Infraestructura } \\
\text { - Comunicación }\end{array}$ & - \\
\hline 4 & $\begin{array}{l}\text { Estudio de la } \\
\text { satisfacción } \\
\text { percibida por los } \\
\text { estudiantes de la } \\
\text { UNED con su vida } \\
\text { universitaria } \\
\text { (González et al., } \\
\text { 2017) }\end{array}$ & 2017 & 60 estudiantes & $\begin{array}{l}\text { - Capacidad } \\
\text { - Docencia } \\
\text { - Contenidos } \\
\text { - Comunicación }\end{array}$ & $\begin{array}{l}67.75 \\
50.16 \\
34.97 \\
26.13\end{array}$ \\
\hline
\end{tabular}




\begin{tabular}{|c|c|c|c|c|c|}
\hline 5 & $\begin{array}{l}\text { Satisfação com a } \\
\text { educação recebidae } \\
\text { rendimento } \\
\text { académico em } \\
\text { estudantes do } \\
\text { ensino superior da } \\
\text { cidade da beira, } \\
\text { moçambique } \\
\text { (Nhantumbo et al., } \\
\text { 2018). }\end{array}$ & 2018 & 400 estudiantes & $\begin{array}{l}\text { Satisfacción } \\
\text { educativa } \\
\text { - General } \\
\text { Desempeño } \\
\text { académico } \\
\text { - Suficiente }\end{array}$ & 64.25 \\
\hline 6 & $\begin{array}{l}\text { Investigating } \\
\text { Determinants of } \\
\text { Student } \\
\text { Satisfaction in the } \\
\text { First } \\
\text { Year of College in a } \\
\text { Public University in } \\
\text { the State of Qatar } \\
\text { (Al-Sheeb et al., } \\
\text { 2018) }\end{array}$ & 2018 & 282 estudiantes & $\begin{array}{l}\text { Satisfacción general } \\
\text { - Sentido de } \\
\text { pertenencia } \\
\text { - Satisfacción en el } \\
\text { aula de clase }\end{array}$ & 77 \\
\hline 7 & $\begin{array}{l}\text { Evaluación de la } \\
\text { calidad de los } \\
\text { servicios. Un caso } \\
\text { de estudio en una } \\
\text { universidad } \\
\text { Ecuatoriana } \\
\text { (Peñaherrera et al., } \\
2019 \text { ) }\end{array}$ & 2019 & $\begin{array}{ll}\text { Estudio } & \text { de } \\
\text { validez } & \end{array}$ & $\begin{array}{l}\text { - Elementos tangibles } \\
\text { - Confiabilidad } \\
\text { - Capacidad de } \\
\text { respuesta } \\
\text { - Seguridad } \\
\text { - Empatía }\end{array}$ & - \\
\hline 8 & $\begin{array}{lr}\text { Calidad } & \text { del } \\
\text { servicio, } & \\
\text { satisfacción } & \text { y } \\
\text { lealtad } & \text { de }\end{array}$ & 2020 & 262 estudiantes & $\begin{array}{l}\text { - Infraestructura } \\
\text { - Capacidad de } \\
\text { servicio }\end{array}$ & $\begin{array}{l}17 \\
21 \\
21 \\
39\end{array}$ \\
\hline
\end{tabular}




\begin{tabular}{|c|c|c|}
\hline $\begin{array}{l}\text { estudiantes } \\
\text { universitarios } \\
\text { peruanos }\end{array}$ & $\begin{array}{l}\text { - Eficiencia del } \\
\text { servicio } \\
\text { - Competencia y } \\
\text { actitudes de los } \\
\text { docentes } \\
\text { - Calidad de servicio }\end{array}$ & 70 \\
\hline
\end{tabular}

Fuente: Elaborado por los autores

En función a los resultados se entiende que las universidades podrían utilizar los datos de satisfacción de los estudiantes para mejorar aún más sus áreas de alto rendimiento, así como para resaltar aquellas áreas que necesitan mejoras. Además, se puede adoptar un enfoque sistemático de la retroalimentación de los estudiantes y actuar rápidamente sobre los resultados podría fortalecer sustancialmente la participación y satisfacción de los estudiantes.

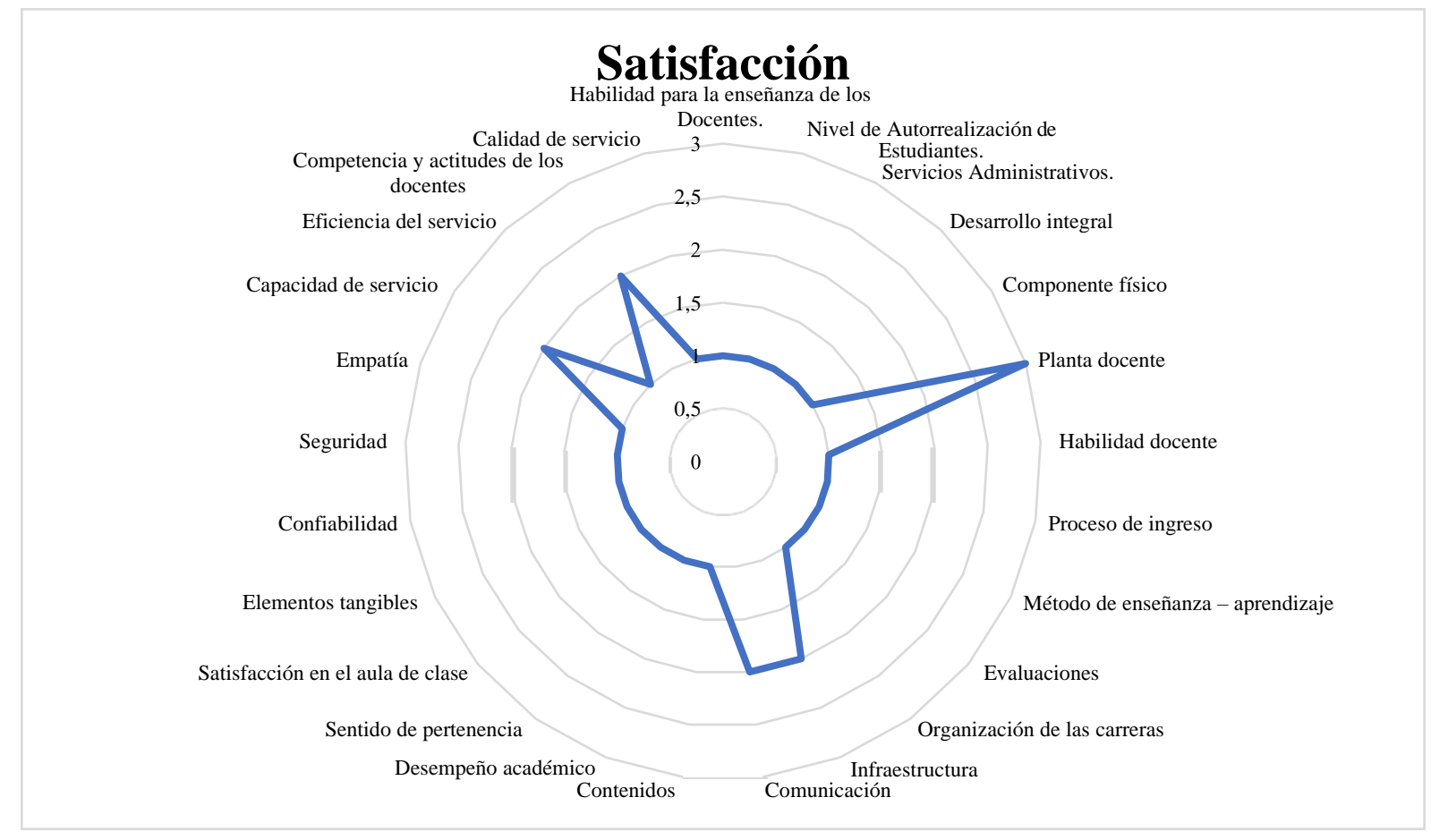

Figura 3. Satisfacción de los servicios universitarios

Fuente: Elaborado por los autores

Bajo los datos encontrados por medio de la revisión bibliográfica, se encuentra que los estudiantes basan su satisfacción principalmente en la planta docente, no obstante, existe aspectos relevantes como son la infraestructura, capacidad de servicio, comunicación y 
competencias y actitudes de los docentes, es relevante mencionar que los estudiantes emiten una opinión de satisfacción basándose en la calidad de los servicios recibidos, sobre todo de los que aportan a tener un mejor desempeño académico.

Tabla 3. Relación entre las estrategias de aprendizaje y la satisfacción de los servicios universitarios

\begin{tabular}{|c|c|c|c|c|c|c|}
\hline \# & Estudio & Año & $\begin{array}{l}\text { Població } \\
\text { n } \\
\text { evaluada }\end{array}$ & Objetivo & Resultado & $\begin{array}{l}\text { Significanci } \\
\text { a }\end{array}$ \\
\hline 1 & $\begin{array}{l}\text { Analysis of } \\
\text { Student } \\
\text { Satisfaction in } \\
\text { The Process of } \\
\text { Teaching } \\
\text { and Learning } \\
\text { Using } \\
\text { Importance } \\
\text { Performance } \\
\text { Analysis } \\
\text { (Sembiring et } \\
\text { al., 2017) }\end{array}$ & $\begin{array}{l}201 \\
7\end{array}$ & $\begin{array}{l}1204 \\
\text { estudiante } \\
\text { s }\end{array}$ & $\begin{array}{l}\text { Determinar } \\
\text { el nivel de } \\
\text { satisfacción } \\
\text { de los } \\
\text { estudiantes } \\
\text { en el proceso } \\
\text { de } \\
\text { aprendizaje. }\end{array}$ & $\begin{array}{l}\text { La experiencia } \\
\text { de los } \\
\text { maestros, los } \\
\text { cursos } \\
\text { ofrecidos, el } \\
\text { entorno de } \\
\text { aprendizaje, } \\
\text { estrategias y } \\
\text { las } \\
\text { instalaciones } \\
\text { del aula } \\
\text { mejoran la } \\
\text { satisfacción de } \\
\text { los estudiantes } \\
\text { en la educación } \\
\text { superior. }\end{array}$ & $\begin{array}{l}\text { Relación } \\
\text { significativa } \\
\text { y positiva }\end{array}$ \\
\hline 2 & $\begin{array}{l}\text { International } \\
\text { students' } \\
\text { satisfaction } \\
\text { with university } \\
\text { services: The } \\
\text { case of } \\
\text { postgraduate } \\
\text { students from }\end{array}$ & $\begin{array}{l}201 \\
8\end{array}$ & $\begin{array}{l}2043 \\
\text { estudiante }\end{array}$ & $\begin{array}{l}\text { Tratar de } \\
\text { llenar la } \\
\text { brecha en la } \\
\text { literatura } \\
\text { científica } \\
\text { con respecto } \\
\text { a la } \\
\text { satisfacción }\end{array}$ & $\begin{array}{l}\text { El análisis } \\
\text { mostró que la } \\
\text { relevancia de } \\
\text { los cursos } \\
\text { académicos y } \\
\text { actividades } \\
\text { sociales como } \\
\text { estrategias de }\end{array}$ & $\begin{array}{l}\text { Existe } \\
\text { relación }\end{array}$ \\
\hline
\end{tabular}




\begin{tabular}{|c|c|c|c|c|c|c|}
\hline & $\begin{array}{l}\text { Central Asia } \\
\text { (Sabateyana et } \\
\text { al., 2018) }\end{array}$ & & & $\begin{array}{l}\text { de los } \\
\text { estudiantes }\end{array}$ & $\begin{array}{l}\text { aprendizaje de } \\
\text { forma implícita } \\
\text { fueron } \\
\text { variables } \\
\text { determinantes } \\
\text { para predecir la } \\
\text { satisfacción } \\
\text { general }\end{array}$ & \\
\hline 3 & $\begin{array}{l}\text { Does e- } \\
\text { learning } \\
\text { service quality } \\
\text { influence e- } \\
\text { learning } \\
\text { student } \\
\text { satisfaction and } \\
\text { loyalty? } \\
\text { Evidence from } \\
\text { Vietnam } \\
\text { (Pham et al., } \\
\text { 2019) }\end{array}$ & $\begin{array}{l}201 \\
9\end{array}$ & $\begin{array}{l}1232 \\
\text { estudiante } \\
\mathrm{s}\end{array}$ & $\begin{array}{l}\text { Examinar las } \\
\text { relaciones } \\
\text { entre los } \\
\text { atributos de } \\
\text { calidad del } \\
\text { servicio de } \\
\text { e-learning, la } \\
\text { calidad de } \\
\text { general del } \\
\text { servicio de } \\
\text { e-learning, la } \\
\text { satisfacción } \\
\text { de los } \\
\text { estudiantes } \\
\text { de e-learning } \\
\text { y la lealtad } \\
\text { de los } \\
\text { estudiantes } \\
\text { de e-learning }\end{array}$ & $\begin{array}{l}\text { Los resultados } \\
\text { indican una } \\
\text { relación } \\
\text { positiva entre } \\
\text { la calidad } \\
\text { general del } \\
\text { servicio de e- } \\
\text { learning y la } \\
\text { satisfacción de } \\
\text { los estudiantes } \\
\text { de e-learning }\end{array}$ & $\begin{array}{l}\text { Relación } \\
\text { positiva }\end{array}$ \\
\hline 4 & $\begin{array}{l}\text { University } \\
\text { student } \\
\text { satisfaction, } \\
\text { resource } \\
\text { management } \\
\text { and }\end{array}$ & $\begin{array}{l}202 \\
0\end{array}$ & $\begin{array}{l}364 \\
\text { estudiante } \\
\text { s }\end{array}$ & $\begin{array}{l}\text { Determinar } \\
\text { la relación } \\
\text { entre la } \\
\text { satisfacción } \\
\text { de los } \\
\text { estudiantes }\end{array}$ & $\begin{array}{l}\text { Los resultados } \\
\text { de la } \\
\text { investigación } \\
\text { muestran que } \\
\text { las estrategias } \\
\text { de aprendizaje }\end{array}$ & $\begin{array}{l}\text { Nivel de } \\
\text { significanci } \\
\text { a baja }\end{array}$ \\
\hline
\end{tabular}




\begin{tabular}{|l|l|l|l|}
\hline metacognitive & & universitario & autorregulador \\
learning & s con la & as \\
strategies.Gam & & universidad & metacognitivas \\
ze Kasalak \& & y el uso de la & y de gestión de \\
Miray & gestión de & recursos \\
Dağyar(Kasala & recursos y & afectan la \\
k \& Dagyar, & estrategias & satisfacción de \\
2020) & de & los estudiantes \\
& aprendizaje & \\
& autorregulad & \\
& o & \\
\end{tabular}

Fuente: Elaborado por los autores

El uso de estrategias de aprendizaje autorregulado por los estudiantes afecta positivamente su satisfacción con la institución que están estudiando. Además, el impacto indirecto de la gestión de recursos en la satisfacción de los estudiantes puede llegar a ser significativo.

Por otra parte, se evidencia la estrategia de aprendizaje electrónico que constituyen la calidad general del servicio de aprendizaje electrónico (e-learning), con fuertes relaciones con la satisfacción, sin embargo, el estudio demostró tener algunas limitaciones. A pesar de no existir gran cantidad de estudios en donde se evidencie una relación entre las variables de estudio, los aspectos existentes evidencian una relación considerable.

\section{DISCUSIÓN}

Los resultados de la revisión emitieron que las estrategias más comunes son las estrategias metacognitivas, la autorregulación y las estrategias de procesamiento y uso de la información, coincidiendo con esto, se entiende que las actividades académicas deben abarcar todo lo que pueda desarrollar e inculcar buenos valores, actitud, carácter y personalidad fuerte, también debe tener en cuenta el entorno de aprendizaje que incluye una buena infraestructura y un servicio de apoyo (Mineiro \& D'Ávila, 2020). Con el fin de obtener nuevos estudiantes y retener a los estudiantes actuales, deben apuntar a mejorar la satisfacción de los estudiantes y reducir la insatisfacción (Abdulssalam, 2017). Esto 
solo se puede lograr si todos los servicios relacionados con la vida académica, como los servicios implícitos, los servicios explícitos y los servicios físicos, deben entregarse con un estándar adecuado.

Los estudios que han utilizado enfoques integrales para la satisfacción de los estudiantes han diseño un instrumento de satisfacción del estudiante para una universidad, instrumento que incluye servicios administrativos y estudiantiles, ambiente estudiantil, los cursos y apoyo a los profesores (Razinkina et al., 2017; Al-Sheeb et al., 2018). Coincidiendo con la opinión de otros autores, este estudio evidencio que varios de las investigaciones utilizadas son de estudios de validez, en dichos aspectos validados predominan los servicios y su calidad. Todo profesor toma una serie de decisiones y realiza una serie de tareas en su trabajo diario que influyen de manera decisiva en el rendimiento de sus alumnos, sin embargo, es necesario que los estudiantes aporten a la gestión académica (Pinos, Ayala y Bonilla, 2018).

Las percepciones de los estudiantes sobre los aspectos académicos, sociales y ambientales de la comunidad universitaria pueden tener un impacto significativo en las experiencias universitarias en general. Ahora comparando con la revisión literaria emitida seencontró que las percepciones de los estudiantes sobre los aspectos académicos, sociales y ambientales, como es el caso de la experiencia de los maestros, los cursos ofrecidos, el entorno de aprendizaje, estrategias y las instalaciones del aula mejoran la satisfacción de los estudiantes en la educación superior. Sobresaliendo la relevancia de los cursos académicos y actividades sociales como estrategias de aprendizaje de forma implícita fueron variables determinantes para predecir la satisfacción general.

Otros estudios han intentado utilizar enfoques más creativos para determinar la relación de las estrategias de aprendizaje con la satisfacción de los estudiantes. Pham et al, (2019) evaluó las experiencias de los estudiantes en relación al e-learning y la satisfacción, en tal sentido los resultados indican una relación positiva. Desde otra opinión, hoy en día a medida que avanza la tecnología, se están integrando al aula nuevos recursos educativos como el ordenador, el proyector, las pizarras interactivas, las tabletas y los teléfonos inteligentes, que están dando lugar a una nueva tendencia pedagógica donde podemos encontrar e-learning, aula invertida, gamificación, juegos serios, laboratorios o laboratorios remotos, espacios de creadores o educación ubicua (Jadán et al., 2020). 
A partir de narrativas, se entiende que los estudiantes universitarios se centraron en la enseñanza y el aprendizaje y el entorno de servicio, a partir de los cuales seidentificaron una serie de nuevos determinantes.

\section{CONCLUSIONES}

Para la mayoría de las áreas académicas, el desarrollo del aprendizaje para la experiencia inicia cuando el aprendiz ingresa a un curso de nivel superior. Los procesos de aprendizaje, para ser eficientes, deben tener en cuenta una serie de aspectos relacionados con la estructura curricular de un curso, las estrategias de aprendizaje de los estudiantes, el entorno de aprendizaje y los resultados del aprendizaje, expresados por la apropiación del discurso académico por parte de los estudiantes en condiciones aceptables.

Promover el éxito académico entre los estudiantes es fundamental para abordar la necesidad de fomentar las competencias de empleabilidad. Los bajos niveles de logro académico en la educación superior, junto con el número creciente de personas que participan en la educación terciaria, representan tendencias cruciales que deben estudiarse para desarrollar prácticas de retención eficientes. Si bien este trabajo ha logrado sus objetivos, es solo una faceta mínima del conocimiento sobre las estrategias de aprendizaje.

Este estudio mostró que, en general, los servicios de las universidades fueron moderados desde la perspectiva de los estudiantes. Esto significa que las universidades tienen la capacidad suficiente para seguir mejorando. Además, se identificó que las actividades académicas son más importantes que las no académicas, los estudiantes son los jueces más importantes de si esto se ha logrado o no, por lo que se deben realizar encuestas de satisfacción de los estudiantes de forma regular.

Muchos estudios se han centrado en cómo factores como las actitudes, las percepciones y los compromisos académicos y sociales de los estudiantes afectan el éxito y la retención de los estudiantes; sin embargo, pocos estudios han intentado explorar la influencia que estos factores tienen sobre la satisfacción de los estudiantes y sus percepciones generales de la experiencia universitaria, además que, tampoco se ha estudiado a profundidad la relación existente entre las estrategias de aprendizaje y la satisfacción de los estudiantes sobre todo con los servicios que brinda el entorno universitario. 


\section{REFERENCIAS BIBLIOGRÁFICAS}

Abdulssalam, A. (2017). The quality of educational services and its effect on students satisfaction an empirical study on students of Alrifaq Private University in Libya. Global Journal of Commerce \& Management Perspective, 6(1), 1-10. https://doi.org/10.24105/gjcmp.6.1.1701

Abella, V., Aüsín, V., Delgado, V., Hortigüela, D., \& Solano, H. (2018). Determinantes de la calidad, la satisfaccion y el aprendizaje percibido de la e-formación del profesorado üníversitario. Revista Mexicana de Investigacion Educativa, 23(78), 733-760. http://scielo.isciii.es/pdf/fem/v22n3/2014-9832-fem-22-3-103.pdf

Al-Sheeb, B., Hamouda, A. M., \& Abdella, G. M. (2018). Investigating Determinants of Student Satisfaction in the First Year of College in a Public University in the State of Qatar. Education Research International. https://doi.org/10.1155/2018/7194106

Alarcón, M., Alcas, N., Alarcón, H., Natividad, J., \& Rodríguez, A. (2019). Use of learning strategies in the university: A case study. Propósitos y Representaciones, 7(1), 10-32. http://www.scielo.org.pe/pdf/pyr/v7n1/en_a02v7n1.pdf

Alvarado, E., Luyando, J. R., \& Picazzo, E. (2015). Perception of students towards the quality of private universities in Monterrey. Revista Iberoamericana de Educacion Superior, 6(17), 58-76. https://doi.org/10.1016/j.rides.2015.10.003

Botello, J., Elena, D., \& Pérez, R. (2014). Estudio de la Satisfacción de los Estudiantes con los Servicios Educativos brindados por Instituciones de Educación Superior del Valle de Toluca Universities of the Toluca. Revista Iberoamericana Sobre Calidad, Eficacia y Cambio En Educación, 13(2), 5-26.

Cadena, M., Mejías, A., Vega, A., \& Vásquez, J. (2015). La satisfacción estudiantil universitaria: análisis estratégico a partir del análisis de factores. Producción y Gestión, 18(1), 9. https://doi.org/10.15381/idata.v18i1.12062

Contreras, A., Pérez, C., Hechenleitner, M., Vaccarezza, G., \& Toirkeins, J. (2019). Satisfacción académica y prácticas pedagógicas percibidas por estudiantes de salud de Chile. Revista de La Fundación Educación Médica, 22(3), 103. https://doi.org/10.33588/fem.223.992

Dos Anjos, E., \& Dos Reis, C. (2019). Satisfacción con estudios, tiración emocional y estrategias de café en estudiantes universitarios en Manaus- Brasil. Revista Internacional de Psicología Del Desarrollo y La Educación, 4(1). https://www.redalyc.org/jatsRepo/3498/349861666013/html/index.html 
González, M., Pino, M., \& Penado, M. (2017). Estudio de la satisfacción percibida por los estudiantes de la UNED con su vida universitaria. Revista Iberoamericana de Educación a Distancia, 20(1), 243. https://doi.org/10.5944/ried.20.1.16377

Gozalo, M., León, B., \& Mendo, S. (2020). Good practices and learning strategies of undergraduate university students. International Journal of Environmental Research and Public Health, 17(6). https://doi.org/10.3390/ijerph17061849

Hoffman, A., Ledesma, R., \& Liporace, M. (2017). Estilos y estrategias de aprendizaje en estudiantes universitarios de Buenos Aires. Revista de Psicologia, 35(2), 535573. https://doi.org/10.18800/psico.201702.006

Jadán, J., Guevara, C., Lara, P., Sanchez, S., Calle, T., Salvador, L., Acosta, P., \& Bonilla, D. (2020). Building hybrid interfaces to increase interaction with young children and children with special needs. Advances in Intelligent Systems and Computing, 959(August 2019), 306-314. https://doi.org/10.1007/978-3-030-20040-4_28

Kasalak, G., \& Dagyar, M. (2020). University student satisfaction, resource management and metacognitive learning strategies. Teachers and Curriculum, 20(1), 179-195. https://doi.org/10.4324/9780203816165-16

Lara, A., Lara, N., Lara, G., \& Bonilla, D. (2018). Aprendizaje basado en problemas como estrategia significativa en la formación académica. Universidad, Ciencia y Tecnología, 142-149. http://scielo.sld.cu/pdf/rc/v14n62/rc066218.pdf

López, P., Gallegos, S., Vilca, G., \& López, M. (2018). Estrategias de aprendizaje en estudiantes universitarios de ciencias sociales: un estudio empírico en la escuela profesional de sociología UNAP.Comuni@cción - Revista de Investigación En $\begin{array}{llll}\text { Comunicación } & y & \text { Desarrollo, } & 9(1),\end{array}$ http://www.scielo.org.pe/pdf/comunica/v9n1/a04v9n1.pdf

Mena, A., Rodríguez, E., Golbach, M., Abraham, G., \& Fernández, A. (2015). Evaluación de estrategias de aprendizaje en estudiantes universitarios y su relación con el rendimiento académico en procesos mediados por TIC. III Jornadas de TIC e Innovación En El Aula, 1-10.

Mineiro, M., \& D’Ávila, C. (2020). Como aprendem estudantes universitários? Estudo de caso sobre estratégias e estilos de aprendizagem. Revista Diálogo Educacional, 20(64), 93-122. https://doi.org/10.7213/1981-416x.20.064.ds05

Mohallem, R. (2016). Estratégias de Aprendizagem em Universitários: avaliação e intervenção [Universidade Sao Francisco]. 
https://www.usf.edu.br/galeria/getImage/427/17279032934113009.pdf

Montero, C., Sierra, B., \& Mendiarrieta, A. (2017). Eficacia de un programa de mejora de las estrategias de aprendizaje en la enseñanza superior. Psicothema, 29(4), 527532. https://doi.org/10.7334/psicothema2016.171

Mora, E., Bonilla, D., Nuñez, A., \& Sarmiento, C. (2018). Inadaptabilidad de los docentes al manejo de plataformas virtuales: Caso EDUCARECUADOR. Revista Conrado, 14(62), 39-43. http://scielo.sld.cu/pdf/rc/v14n62/rc066218.pdf

Nhantumbo, D., Boza, Á., \& Bruce, B. (2018). Satisfação com a educação recebida e rendimento académico em estudantes do Ensino Superior da Cidade da Beira, Moçambique. Revista Científica Electrónica de Educación y Comunicación En La Sociedad Del Conocimiento, 18(2), 316-334. https://doi.org/10.30827/eticanet.v2i18.11894

Pedro, E., Mendes, L., \& Lourenço, L. (2018). Perceived service quality and students' satisfaction in higher education: The influence of teaching methods. International Journal for Quality Research, 12(1), 165-192.https://doi.org/10.18421/IJQR12.0110

Pegalajar, M. (2016). Estrategias de aprendizaje en alumnado universitario para la formación presencial y semipresencial. Revista Latinoamericana de Ciencias Sociales, Niñez $\quad y \quad$ Juventud, 14(1), 659-676. https://doi.org/10.11600/1692715x.14145071114

Peñaherrera, I., Salinas, E., \& D’Armas, M. (2019). Evaluación de la calidad de los servicios. Un caso de estudio en una universidad Ecuatoriana. Universidad, Ciencia y Tecnolo, 23(90), 4-13. https://uctunexpo.autanabooks.com/index.php/uct/article/view/97/97

Pham, L., Limbu, Y., Bui, T., Nguyen, H., \& Pham, H. (2019). Does e-learning service quality influence e-learning student satisfaction and loyalty? Evidence from Vietnam. International Journal of Educational Technology in Higher Education, 16(1). https://doi.org/10.1186/s41239-019-0136-3

Pinos, G., Ayala, D., \& Bonilla, D. (2018). Desarrollo del pensamiento lógicomatemático a través de juegos populares y tradicionales en niños de educación inicial. Revista Científica Ciencia y Tecnología, 18(19), 133-141. https://doi.org/10.47189/rcct.v18i19.190

Poce, J., \& Gamarra, C. (2014). Estrategias de Aprendizaje y Rendimiento Académico 
En Estudiantes Universitarios de Pregrado. Ágora Revista Científica, 1(2), 25-30. http://www.revistaagora.com/index.php/cieUMA/article/view/17/17

Razinkina, E., Pankova, L., Trostinskaya, I., Pozdeeva, E., Evseeva, L., \& Tanova, A. (2017). Student satisfaction as an element of education quality monitoring in innovative higher education institution. E3S Web of Conferences, 33. https://doi.org/10.1051/e3sconf/20183303043

Ríos, N., Arellano, A., López, V., \& Burgos, A. (2017). Procesos y servicios universitarios: La percepción de estudiantes. Revista de Docencia e Investigación Educativa, $3(8)$, 64-76. http://www.ecorfan.org/spain/researchjournals/Docencia_e_Investigacion_Educati va/vol3num8/Revista_de_Docencia_e_Investigacion_Educativa_V3_N8_7.pdf

Rojo, M., \& Bonilla, D. (2020). COVID-19: La necesidad de un cambio de paradigma económico y social. CienciAmérica, 9(2), 77. https://doi.org/10.33210/ca.v9i2.288

Sabateyana, B., Saduov, A., Madiyarova, E., Jempeissova, G., Selezneva, I., \& Fursova, T. (2018). International students' satisfaction with university services: The case of postgraduate students from Central Asia. Revista Espacios, 39(9). https://www.revistaespacios.com/a18v39n09/a18v39n09p04.pdf

Salazar, I., \& Heredia, Y. (2019). Estrategias de aprendizaje y desempeño académico en estudiantes de Medicina. Educacion Medica, 20(4), 256-262. https://doi.org/10.1016/j.edumed.2018.12.005

Salinda, I., \& Lalitha, F. (2017). Students' Satisfaction in Higher Education Literature Review. American Journal of Educational Research, 5(5), 533-539. https://doi.org/10.12691/education-5-5-9

Sembiring, P., Sembiring, S., Tarigan, G., \& Sembiring, O. (2017). Analysis of Student Satisfaction in the Process of Teaching and Learning Using Importance Performance Analysis. Journal of Physics: Conference Series, 930(1), 0-8. https://doi.org/10.1088/1742-6596/930/1/012039

Tumino, M., \& Poitevin, E. (2014). Evaluación de la calidad de servicio universitario desde la percepción de estudiantes y docentes: caso de estudio. Revista Electrónica Iberoamericana Sobre Calidad, Eficacia y Cambio En Educación, 12(2), 63-84. https://dialnet.unirioja.es/descarga/articulo/4679055.pdf

Vera, A., Poblete, S., \& Días, C. (2018). Percepción de estrategias y estilos de aprendizaje en estudiantes universitarios de primer año. Psicothema, 1-23. https://web-a- 
ebscohost-com.wdg.biblio.udg.mx:8443/ehost/detail/detail?vid=3\&sid=6bf30e69-

c101-47cb-932c-

8cce784b400a\%40sessionmgr4006\&bdata=JnNpdGU9ZWhvc3QtbG12ZQ\%3D\%3

D\#AN=135788438\&db=zbh\%0Ahttps://web-a-ebscohost-

com.wdg.biblio.udg.mx:8443/ehost/pdfvie

\section{PARA CITAR EL ARTÍCULO INDEXADO.}

Arellano, J., Vásconez Torres, M. J., \& Ladino Pazmiño, N. A. (2021). Estrategias de aprendizaje en la virtualidad y la satisfacción de los servicios Universitarios: una revisión Literaria. Magazine De Las Ciencias: Revista De Investigación E Innovación, 6(2), 1-24. https://doi.org/10.33262/rmc.v6i2.1087 